\title{
Angiotensin II receptor blockers and cardiovascular protection: Focus on left ventricular hypertrophy regression and atrial fibrillation prevention
}

\author{
Cesare Cuspidi',2 \\ Francesca Negri ${ }^{2}$ \\ Alberto Zanchetti ${ }^{3}$ \\ 'Department of Clinical Medicine and \\ Prevention, University of Milano- \\ Bicocca, Milan, Italy; ${ }^{2}$ Policlinico di \\ Monza; ${ }^{3}$ Centro Interuniversitario \\ di Fisiologia Clinica e Ipertensione, \\ Università di Milano, and Istituto \\ Auxologico Italiano, Milan, Italy
}

\begin{abstract}
Left ventricular hypertrophy (LVH) and atrial fibrillation (AF) are strong predictors of cardiovascular (CV) morbidity and mortality, independently of blood pressure levels and other modifiable and nonmodifiable risk factors. The actions of circulating and tissue angiotensin II, mediated by AT1 receptors, play an important role in the development of a wide spectrum of cardiovascular alterations, including LVH, atrial enlargement and AF. Growing experimental and clinical evidence suggests that antihypertensive drugs may exert different effects on LVH regression and new onset AF in the setting of arterial hypertension. Since a number of large and adequately designed studies have found angiotensin II receptor blockers (ARBs) to be more effective in reducing LVH than beta-blockers and data are also available showing their effectiveness in preventing new or recurrent $\mathrm{AF}$, it is reasonable to consider this class of drugs among first line therapies in patients with hypertension and LVH (a very high risk phenotype predisposing to AF) and as adjunctive therapy to antiarrhythmic agents in patients undergoing pharmacological or electrical cardioversion of AF.
\end{abstract}

Keywords: angiotensin II receptor blockers, left ventricular hypertrophy, atrial fibrillation

\section{Introduction}

Patients with hypertension are at increased risk of developing a variety of cardiac structural and functional changes, such as increased left ventricular (LV) mass, LV systolic and diastolic dysfunction, impairment of coronary reserve, left atrial and aortic root enlargement, prolonged ventricular repolarization, and arrhythmias (Leonetti et al 1995; Sega et al 2001; Mitchell et al 2007).

Most attention has been focused on LV hypertrophy (LVH), because of the high prevalence of this phenotype and its association with an increased risk of cardiovascular morbidity and mortality. Longitudinal observational studies performed in general population samples and in hypertensive groups have shown that LVH is a robust, independent predictor of sudden death, coronary artery disease, acute cerebrovascular events, and congestive heart failure (Levy et al 1990; Koren et al 1991; Verdecchia et al 2001). A large body of evidence indicates that effective antihypertensive treatment can induce LVH regression (Klingbeil et al 2003), improve systolic and diastolic LV function (Trimarco et al 1988; Perlini et al 2001) as well as reduce ventricular and supra-ventricular arrhythmias (Hennersdorf et al 2001). Reversal of LVH has been shown to be associated with a substantial decrease in fatal and non fatal cardiovascular complications, including new onset atrial fibrillation (AF) (Okin et al 2006).

Evidence is accumulating that the renin - angiotensin system (RAS) in addition to the hemodynamic load plays a pivotal role in the development of hypertensive
Policlinico di Monza,Via Amati I I I, 20052

Monza, Italy

Email cesare.cuspidi@unimib.it 
myocardial hypertrophy and its sequelae. Accordingly, the aim of this review is to discuss the current status of knowledge concerning the cardioprotective effects of angiotensin II receptor blockers (ARBs), focusing on their ability to reverse $\mathrm{LVH}$ and prevent AF.

\section{The RAS, hypertensive LVH, and AF}

Although the pathogenesis of LVH in arterial hypertension is not yet fully understood, several lines of experimental and clinical evidence indicate that haemodynamic stress (ie, pressure and volume overload) is fundamental to the development of LVH; a host of nonhemodynamic factors, however, substantially contribute to modulating the hypertrophic response (Schmieder 2005). In particular, activation of the RAS as a consequence of myocardial stretch and other stimuli is recognized to play a relevant role. In addition to its function in regulating blood pressure (BP), angiotensin II, the active component of RAS, by acting on type 1 receptors has been shown to stimulate various growth factors inducing myocyte hypertrophy and myocardial fibrosis. Although RAS was initially described as a circulating system originating in the kidney, many of its components have been also localized in tissues such as the heart and blood vessels, where they may exert direct effects on cardiomyocyte and noncardiomyocyte cells, endothelial and vascular smooth muscle cells. Angiotensin II both directly and indirectly through aldosterone secretion, has been shown to stimulate fibroblast proliferation, accelerate the turnover of fibrillar collagen, and facilitate deposition of collagen fibers (Campbell et al 1995). Overall, these processes tend to alter tissue structure and increase myocardial stiffness leading to diastolic dysfunction, tachyarrhythmias and ultimately systolic dysfunction. In hypertensive patients a significant correlation has been shown between the circulating levels of angiotensin II (but not plasma renin activity or angiotensin I) and several echocardiographic indices of LVH or LV concentric remodelling. These findings result from a number of studies showing that patients with inappropriately high angiotensin II concentrations in relation to dietary sodium intake have a more pronounced LV involvement than their counterparts with relatively low angiotensin II levels (Schmieder et al 1996). Furthermore, an association has been reported between angiotensin II and LV mass, independently of ambulatory blood pressure, body mass index and sodium excretion values (Schmieder et al 1988; Schlaich et al 1998). Finally, the LVH phenotype appears to correlate with the presence of vascular remodelling and hypertrophy, atherosclerotic lesions, increased coagulation and reduced fibrinolysis, namely a series of phenomena also associated with RAS activation.

Activation of RAS is also thought to be involved in the pathophysiology of AF, and recent data from intervention trials suggest that inhibition of RAS might have a role in the prevention of AF and its complications. The development and persistence of AF are both associated with changes in cardiac structure, function and electrical properties known as cardiac remodeling. Angiotensin II type 1 receptor stimulation induces atrial myocyte hypertrophy, fibrosis and affects atrial contractility leading to atrial structural remodelling (Kaschina et al 2003), on the other hand it has been suggested that angiotensin II type 2 stimulation counteracts this effect (Rogg et al 1996), by inhibiting proliferative processes. Structural remodeling in patients with chronic AF is characterized by a progressive atrial enlargement and fibrosis, with histological evidence of a large amount of connective tissue between atrial myocytes, leading to intra-atrial conduction disturbances and persistent electrophysiological inhomogeneities. Angiotensin II might also alter atrial electrophysiology by modifying ion exchanges within atrial cells: angiotensin type 1 receptor stimulation activates phospholipase $\mathrm{C}$, and, consequently, via the inositol-triphosphate releases calcium from sarcoplasmatic reticulum, the resulting blunted outward potassium current leads to increased chamber refractoriness (Choudhury et al 2005). Furthermore, polymorphisms of RAS gene have been reported to play a role in predisposing patients to AF (Tsai et al 2004). Finally, angiotensin II has proinflammatory and prothrombotic properties that might concur to induce a prothrombotic status in AF. The strongest argument, however, for a critical role of RAS in AF comes from recent clinical trials showing that RAS blockade prevents new onset as well as recurrence of AF in different clinical settings.

\section{LVH regression: Clinical aspects and role of ARBs}

The prevalence of echocardiographic LVH is dependent on the criteria used for the diagnosis (gender and nongender specific, indexed to body surface area or height, more or less restrictive) and more importantly on clinical and demographic characteristics of the patients examined. In uncomplicated never-treated hypertensives the prevalence rates of $\mathrm{LVH}$ have been reported to range from $15 \%$ to $30 \%$ in studies conducted in population-based samples and in selected groups of patients referred to specialist centres (Sega et al 2001; Cuspidi et al 2002). Higher rates of LVH or LV concentric remodeling have been reported in the same 
settings when other risk factors (ie, metabolic syndrome, obesity) were associated to BP elevation (Cuspidi et al 2004). In treated hypertensives, the prevalence of LVH has been reported to span over a very wide range $(10 \%-70 \%)$ : the prevalence is indeed strictly related to clinic and even more to ambulatory BP control, to the presence of the abovementioned clinical conditions, or to associated $\mathrm{CV}$ diseases (ie, peripheral artery disease, renal insufficiency).

Sustained BP lowering obtained through nonpharmacologic and pharmacologic treatment is usually associated with substantial reductions in LV mass. Regression of LVH during antihypertensive therapy is more closely related with the changes in ambulatory BP than with those in office $\mathrm{BP}$, since the former better reflects the overall BP load to which the LV is exposed during daily life (Mancia et al 1997). Thus, reversing LVH is now regarded as an important intermediate end-point reflecting the protective impact of the antihypertensive therapy, since serial changes in LV mass have been repeatedly shown to positively correlate with fatal and nonfatal $\mathrm{CV}$ outcomes in long-term prospective observational trials (Verdecchia et al 2003). More direct information on this topic has been recently obtained in the LIFE echocardiographic substudy, involving 960 patients with electrocardiographic LVH. At the end of the 5-year study, a decrease of $25 \mathrm{~g} / \mathrm{m}^{2}$ (ie, one standard deviation) of the LV mass index was associated with a $20 \%$ reduction of the primary end-point, after adjusting for several potential confounders such as type of treatment, basal and treatment BP, and basal LV mass index (Devereux et al 2004).

Much of the information on the efficacy of one drug class over another in decreasing LV mass in hypertensive patients is based on a series of meta-analyses published between 1992 and 2003 (Dahlof et al 1992; Cruickshank et al 1992; Schmieder et al 1996; Schmieder et al 1998; Klingbeil et al 2003). A common feature of all these meta-analyses is that they included only echocardiographic studies performed in selected hypertensive subjects with preserved LV systolic function, without valvular heart disease, cardiomyopathy and underlying coronary heart disease. Four meta-analyses have suggested that angiotensin-converting enzyme (ACE) inhibitors are more effective than other antihypertensive agents in reducing $\mathrm{LVH}$, for similar reductions in BP.

However, the most updated meta-analysis indicates that ARBs, ACE-inhibitors, and calcium antagonists are all more effective in reversing $\mathrm{LVH}$ than beta-blockers, while the efficacy of diuretics is intermediate (Klingbeil et al 2003). All meta-analysis findings must be considered with caution as many studies included were noncomparative or nonrandomized, and often of too small a size. For example, of the 109 studies analysed by Dahlof and colleagues (1992) less than one fifth were randomized, double blind, parallel group comparisons. Klingbeil and colleagues (2003) included only double blind, randomized, controlled and parallel studies in their meta-analysis, however, some of them were small (7 patients per treatment arm) and of short duration (4 or more weeks).

Because of such limitations meta-analyses cannot offer incontrovertible evidence of advantages of specific drug classes. Thus, more reliable information is provided by a few large and rigorously designed trials performed in the last decade. The findings of these studies indicate that: 1) BP lowering by whatever agent or agent combination is associated with reduction of an increased LV mass; 2) ARBs, ACE-inhibitors, and calcium antagonists are similarly effective, and more effective than other drug classes. It has been speculated that the marked effectiveness of ARBs in reversing $\mathrm{LVH}$ is related to a more potent and selective RAS inhibition. ARBs, indeed, interfere with both ACE and nonACE generated angiotensin II. Due to their pharmacological properties, they selectively interact with the AT1 receptors only, leaving unaffected the AT2 receptors, whose supposed antiproliferative actions may be stimulated by the increased levels of circulating angiotensin II. Although recent evidence obtained in genetically modified experimental animals does not support a direct role of cardiac RAS in LVH (Reudelhuber et al 2007), it should be also noted that these findings do not rule out a direct role for angiotensin II in cardiac remodeling when combined with other humoral, mechanical, or pathological stimuli.

To date, more than 60 echocardiographic trials have evaluated the effects of the ARBs on LVH in hypertensive patients, showing that the selective blockade of AT1 receptors results in significant reductions of LV mass. Among 24 trials reviewed by Dahlof and colleagues (2001) including a total of 907 patients, only two failed to show significant effects of ARBs on LVH regression. Unfortunately, only few studies in Dahlof's review fulfilled rigorous methodological criteria, as only 11 of them followed a randomized double blind design, three lasted more than six months and three included at least 50 patients per treatment arm.

In this review, we will discuss only the six trials that satisfied all the above mentioned criteria; keeping in mind, however, that the number of 50 patients per treatment-arm is less than that required to detect small but clinically relevant between-treatment differences in LV mass. Overall, these studies involved 1873 patients, randomized to 12 treatment 
Table I Randomized studies comparing the effects of ARBs on LV mass in hypertensive patients

\begin{tabular}{|c|c|c|c|c|}
\hline $\begin{array}{l}\text { Author } \\
\text { reference }\end{array}$ & Drugs & $\begin{array}{l}\text { Study } \\
\text { population } \\
\text { (number) }\end{array}$ & $\begin{array}{l}\text { Duration } \\
\text { (months) }\end{array}$ & $\begin{array}{l}\text { LV mass } \\
\text { decrease } \\
\left(\mathrm{g} / \mathrm{m}^{2}\right)\end{array}$ \\
\hline \multirow[t]{2}{*}{ Malmqvist et al 2000} & Irbesartan & 114 & 11 & -26 \\
\hline & Atenolol & & & -14 \\
\hline \multirow[t]{2}{*}{ Dahlof et al $200 \mathrm{I}$} & Losartan & 225 & 9 & -6.5 \\
\hline & Atenolol & & & -3.7 \\
\hline \multirow[t]{2}{*}{ Cuspidi et al 2002} & Candesartan & 196 & 10 & -15.0 \\
\hline & Enalapril & & & -13.1 \\
\hline \multirow[t]{2}{*}{ Schneider et al 2004} & Irbesartan & 240 & 18 & -8.4 \\
\hline & Atenolol & & & -3.3 \\
\hline \multirow[t]{2}{*}{ Yasunary et al 2004} & Valsartan & 104 & 8 & -16.0 \\
\hline & Amlodipine & & & -1.2 \\
\hline \multirow[t]{2}{*}{ Devereux et al 2004} & Losartan & 960 & 60 & -21.7 \\
\hline & Atenolol & & & -17.7 \\
\hline
\end{tabular}

arms: $\operatorname{losartan}(n=2)$, irbesartan $(n=2)$, candesartan $(n=1)$, valsartan $(n=1)$, amlodipine $(n=1)$, enalapril $(n=1)$, and atenolol $(n=4)$. One study evaluating the effect of irbesartan and atenolol treatments during 11 months in 114 patients with LVH (LV mass index $>131 \mathrm{~g} / \mathrm{m}^{2}$ in men and $>100 \mathrm{~g} / \mathrm{m}^{2}$ in women) showed that both agents progressively reduced LV mass index, the reduction being significantly greater in the irbesartan group (16\% vs $9 \% \mathrm{p}<0.01)$ (Malmqvist et al 2000), as well as the proportion of patients with normalized LV mass (47\% vs 32\%). The REGAAL study was a 36-week multi-centre study comparing the effect of losartan and atenolol (50 mg/day, with possible titration to $100 \mathrm{mg} /$ day and addition of hydrochlorothiazide) in 225 patients with LV mass $>120 \mathrm{~g} / \mathrm{m}^{2}$ and $105 \mathrm{~g} / \mathrm{m}^{2}$ in men and women, respectively (Dahlof et al 2002). Losartan-based regimen significantly reduced LV mass index from baseline $\left(-6.6 \mathrm{~g} / \mathrm{m}^{2}, \mathrm{p}<001\right)$, whereas atenolol had no significant effect $\left(-3.7 \mathrm{~g} / \mathrm{m}^{2}, \mathrm{p}=\mathrm{ns}\right)$. The CATCH, a European multicentre study designed to compare the effects of candesartan and enalapril in 239 hypertensives with increased LV mass ( $>120 \mathrm{~g} / \mathrm{m}^{2}$ in men and $100 \mathrm{~g} / \mathrm{m}^{2}$ in women), demonstrated that candesartan and enalapril reduced LV mass index to a similar extent (by $15.0 \mathrm{~g} / \mathrm{m}^{2}$ and $13.1 \mathrm{~g} / \mathrm{m}^{2}$, respectively) in comparison to baseline (Cuspidi et al 2002). At the end of 48-week treatment, the proportion of patients achieving normalization of LV mass index was higher with candesartan (36\% vs $28 \%$ ), although the difference did not achieve statistical significance. In the CardioVascular Irbesartan Project 240 patients with essential hypertension (32\% of men and $42 \%$ of women having $\mathrm{LVH}$, according to sex-specific criteria of $134 / 110 \mathrm{~g} / \mathrm{m}^{2}$ ) were treated with irbersartan and atenolol for 18 months. A significant reduction in LV mass index was observed only in subjects within the highest quartile of baseline LV mass in the irbesartan $\left(-8.4 \mathrm{~g} / \mathrm{m}^{2}\right)$ but not in the atenolol-treated arm $\left(-3.3 \mathrm{~g} / \mathrm{m}^{2}\right)$ (Schneider et al 2004). Yasunary and colleagues (2004) evaluated the effect of valsartan and amlodipine on LV mass index and inflammatory markers (reactive oxygen species, C-reactive protein) in 104 patients with hypertension and $\mathrm{LVH}$ (according to the sex specific index of 134/110 g/m²) over a period of eight months. Despite similar effects on $\mathrm{BP}$, significant differences on LV mass index were found between valsartan and amlodipine treatment (mean decrease $16.0 \mathrm{~g} / \mathrm{m}^{2}$ vs $1.2 \mathrm{~g} / \mathrm{m}^{2}$, respectively) as well as on inflammatory markers (Yasunary et al 2004). The echocardiographic LIFE substudy was designed to test the ability of losartan to reduce LV mass more than atenolol over a period of 5 years (Devereux et al 2004). Blinded readings of echocardiograms in 457 loasartantreated and 459 atenolol-treated participants were used in an intention-to-treat analysis. Losartan-based therapy induced a greater reduction in LV mass index from baseline to the last available echocardiogram than atenolol after adjustment for baseline LV mass index and BP and in-treatment BP (-21.7 vs $\left.-17.7 \mathrm{~g} / \mathrm{m}^{2}, \mathrm{p}=0.02\right)$. Greater LV mass reductions with losartan were observed in different subgroups: with mild or more severe baseline LVH, younger ( $<65$ years) and older participants, women and men.

\section{Prevention of AF and ARBs}

$\mathrm{AF}$ is the most common cardiac arrhythmia and is strongly associated with a substantial risk of stroke and thromboembolism. The prevalence of AF increases dramatically with advancing age, from $0.1 \%-0.2 \%$ in subjects aged less than 50 years to $9 \%-10 \%$ in those over 80 years. AF often coexists with hypertension, coronary heart disease, valvular heart disease, heart failure, diabetes mellitus and obesity. Currently, hypertension is the most prevalent, independent and potentially modifiable risk factor for AF on a population basis (Kannel et al 1998). Hypertension may lead to AF through left atrial enlargement as a consequence of increased intra-atrial pressure driven by reduced LV compliance and LVH. Increased LV mass and enlargement of left atrium have been identified as independent determinants of new onset AF (Verdecchia et al 2003). In population-based studies, AF has been associated with a 5-2 times higher risk of death compared with subjects free of AF, adjusting for associated cardiac risk factors and cardiac disease, and as much as 2.5 time the risk of death in hypertensive subjects with LVH.

A large interest has been created by recent studies indicating a lower incidence of new and recurrent AF in 
Table 2 Randomized studies comparing the effects of ARBs on new-onset or recurrence of AF in different clinical settings

\begin{tabular}{|c|c|c|c|c|}
\hline $\begin{array}{l}\text { Author } \\
\text { reference }\end{array}$ & Drugs & $\begin{array}{l}\text { Study population } \\
\text { (number) and setting }\end{array}$ & $\begin{array}{l}\text { Duration } \\
\text { (months) }\end{array}$ & $\begin{array}{l}\text { Incident } \\
\text { AF (\%) }\end{array}$ \\
\hline \multirow[t]{2}{*}{ Watchtell et al 2005} & Losartan & 8.851 & 56 & 3.48 \\
\hline & Atenolol & HTN and LVH & & 5.83 \\
\hline \multirow[t]{2}{*}{ Schmieder et al 2006} & Valsartan & 14849 & 50 & 3.67 \\
\hline & Amlodipine & High risk HTN & & 4.34 \\
\hline \multirow[t]{2}{*}{ Ducharme et al 2006} & Candesartan & 6446 & 38 & 5.55 \\
\hline & Placebo & $\mathrm{CHF}$ & & 6.74 \\
\hline \multirow[t]{2}{*}{ Maggioni et al 2005} & Valsartan & 4395 & 23 & 6.53 \\
\hline & Placebo & $\mathrm{CHF}$ & & 7.95 \\
\hline \multirow[t]{2}{*}{ Madrid et al 2002} & Irbesartan & 154 & 8.5 & II.3 \\
\hline & Placebo & AF/Cardioversion & & 29.3 \\
\hline \multirow[t]{2}{*}{ Fogari et al 2006} & Losartan & 250 & 12 & 13 \\
\hline & Amlodipine & $\mathrm{HTN}+\mathrm{AF}$ & & 39 \\
\hline
\end{tabular}

patients treated with ACE-inhibitors or ARBs. The beneficial effect of ARBs and ACE-inhibitors in preventing new onset or recurrent $\mathrm{AF}$ is possibly related to multiple mechanisms. Hypertension and heart failure are both associated with RAS activation and LV structural alterations; it has been shown that treatment of these conditions with ARBs or ACE-inhibitors may reduce the risk of AF by preventing or reversing LVH and atrial enlargement in the setting of arterial hypertension and by slowing the progression of cardiac remodeling in heart failure. This is an exciting novel approach to primary and secondary prevention of AF, because these drugs, beyond and over their BP lowering effect in subjects with hypertension, may act on the underlying cardiac structural and electrical abnormalities that lead to AF.

Post-hoc analyses of two large hypertension (LIFE and VALUE) and two heart failure (CHARM and Val-HeFT) trials have reported beneficial effects of ARBs on new onset AF. The demonstration of favorable effects of these agents on AF relapse, however, is based on three small prospective studies and on a retrospective analysis of data from ACE-inhibitor- or ARB-treated patients with chronic heart failure (CHF) or impaired LV function.

In a post-hoc analysis of the LIFE study including a total of 8.851 patients on sinus rhythm at the baseline electrocardiogram and followed-up for 4.8 years, new onset AF was identified by annual in-study ECGs, analysed in a core centre, in 150 patients randomized to losartan versus 221 patients randomized to atenolol (Wachtell et al 2005); this resulted in a $33 \%$ relative risk reduction $(p<0.001)$ of new onset AF between treatment groups, independent of several confounders. Adjustment for difference in ECG LVH and Framingham risk score had minimal effects on the lower incidence of new AF associated with losartan. Furthermore, patients taking losartan tended to stay on sinus rhythm for a longer period from baseline than those on atenolol (1809 vs 1709 days, $\mathrm{p}=0.05$ ).

A pre-specified objective of the VALUE trial was to compare the effects of valsartan and amlodipine on new onset AF (ie, the incidence of either persistent or at least one occurrence of AF) (Schmieder et al 2006). On ECG recordings obtained every year, and centrally analyzed for ECG-LVH and AF, the incidence of at least one documented occurrence of new onset AF was $3.67 \%$ in the valsartan and $4.34 \%$ in the amlodipine treated-arm (odds ratio 0.84 , $\mathrm{p}=0.011$ ). Taking into account potential confounding factors as covariates (age, history of coronary artery disease, LVH) the incidence of AF persisted significantly lower in the valsartan treated-arm.

In the CHARM program, a large double blind trial aimed at evaluating the effects of candesartan on cardiovascular mortality and morbidity in a broad spectrum of patients with symptomatic chronic heart failure, the incidence of new AF was a pre-specified secondary outcome (Ducharme et al 2006). Of the 6446 patients on normal sinus rhythm at baseline ECG, 392 (6.08\%) developed AF during follow-up, $177(5.55 \%)$ in the candesartan group and $215(6.74 \%)$ in the placebo group, with a relative risk reduction of $19.2 \%$, after adjustment for baseline covariates.

The occurrence of AF, based on adverse event reports, was evaluated post-hoc in patients with heart failure, randomized to valsartan or placebo on top of their prescribed treatments, enrolled in the Val-HeFT trial (Maggioni et al 2005). During the mean 23 months of follow-up, AF was reported in $287 / 4395(6.53 \%)$ on sinus rhythm at baseline, of whom $113 / 2205$ were allocated to valsartan, and 174/2190 (7.95\%) to placebo $(-37 \%, \mathrm{p}=0.0002)$. Multivariate analyses showed that brain peptide levels at baseline, age over 70, male gender and the valsartan treatment were independently associated 
with AF occurrence. In addition to the previous studies in selected patient samples with heart failure and hypertension, other studies have examined the effectiveness of RAS inhibition in maintaining sinus rhythm. A prospective, randomized study including 154 patients with persistent AF, showed that addition of irbesartan (150-300 mg/day) to amiodarone, 3-6 weeks prior to electrical cardioversion, resulted in a lower rate of recurrence of $\mathrm{AF}$ than amiodarone alone (Madrid et al 2002). In a recent study 250 mild hypertensives on sinus rhythm and two documented episodes, at least, of symptomatic AF in the previous 6 months and on treatment with amiodarone $(200 \mathrm{mg} /$ day $)$ were randomized to losartan (50-150 mg/day) and amlodipine (5-10 mg/day) and were followed up for 1 year (Fogari et al 2006): at least one ECG-documented episode of AF was observed in 13\% of the patients treated with losartan and in $39 \%$ of those treated with amlodipine $(p<0.001)$.

Finally, in a meta-analysis, including 11 studies (seven with ACE-inhibitors and four with ARBs) and 56,308 patients from different clinical settings, namely heart failure (4), hypertension (3), cardioversion after AF (2), and myocardial infarction (2) reduction of AF was similar between the two class of drugs (ACE-inhibitors $-28 \%$, $\mathrm{p}=0.001$, ARBs $-29 \%, \mathrm{p}=0.0002$ ) (Healey et al 2005).

Although recent data on ARBs and AF have generated well justified interest, it must be admitted that there are some limitations in the available evidence. Most of the analyses have been post-hoc or used AF as secondary or tertiary endpoint. AF has been searched on electrocardiograms recorded at lengthy intervals. In hypertension trials, a placebo-arm was not allowed: it is, therefore, difficult to conclude whether ARBs did really reduce new onset AF or the comparative treatment did increase the chance of AF. The two CHF studies used placebo, but in the context of multiple and variable background therapy which represented an uncontrollable confounding factor. The studies on recurrent AF were apparently designed ad hoc, but were too small to provide a definite answer. No comparative data are yet available, so far, about a direct comparison between ARBs and ACEIs. It should be finally underlined that specific trials on AF as primary endpoint are ongoing and more direct evidence on this issue may be available soon (Disertori et al 2006).

\section{Conclusion}

Either LVH and AF are strong predictors of cardiovascular events, independently of coexistent modifiable and nonmodifiable risk factors. The effects of angiotensin II, mediated through the AT1 receptor, are critical in the development of cardiac structural and functional alterations in combination with humoral, mechanical and pathological stimuli.

A growing amount of experimental and clinical data suggest that antihypertensive drugs may exert different effects on $\mathrm{LVH}$ regression and on new onset AF in the setting of arterial hypertension. Although more direct evidence on the role of ARBs on AF is desirable (and hopefully will become available soon), it is reasonable to consider this class of drugs among first line therapies in patients with hypertension and LVH (a very high risk phenotype predisposing to $\mathrm{AF}$ ) and as adjunctive therapy to antiarrhythmic agents in patients undergoing pharmacological or electrical cardioversion of AF. This position has also been taken by the recent ESH/ESC guidelines on arterial hypertension.

Although the association of an ACE-inhibitor and angiotensin receptor antagonist has been reported to exert a more pronounced antiproteinuric effect than either component alone in diabetic and nondiabetic nephropathy, favorable evidence for its use in patients with LVH is lacking, so far. The advantages of the full blockade of RAS in reversing $\mathrm{LVH}$ and preventing AF, as predefined secondary end points, is at the present time under investigation in the ONTARGET, an international trial comparing an ARB with an ACE-inhibitor and their combination in high risk individuals.

\section{Declaration of interest}

The authors have no conflicts of interest to report.

\section{References}

Campbell JE, Janicki JS, Weber KT. 1995. Temporal differences in fibroblast proliferation and phenotype expression in response to chronic administration of angiotensin II or aldosterone. $\mathrm{J} \mathrm{Mol} \mathrm{Cell} \mathrm{Cardiol,}$ 27:1545-60.

Choudhury A, Varughese G, Lip GYH. 2005. Targeting the renin angiotensin aldosterone system in atrial fibrillation: a shift from electrical to structural therapy? Exp Opin Pharmacother, 6:2193-207.

Cruickshank JM, Lewis J, Moore V, et al. 1992 Reversibility of left ventricular hypertrophy by different types of antihypertensive therapy. $J$ Hum Hypertens, 6:85-90.

Cuspidi C, Ambrosioni E, Mancia G, et al. 2002. Role of echocardiography and carotid ultrasonography in stratifying risk in patients with essential hypertension: the Assessment of Prognostic Risk Observational Survey. J Hypertens, 20:1307-14.

Cuspidi C, Meani S, Fusi V, et al. 2004. Metabolic syndrome and target organ damage in untreated essential hypertensives. J Hypertens, 22:1991-8

Cuspidi C, Muiesan ML, Valagussa L, et al. 2002. Comparative effects of candesartan and enalapril on left ventricular hypertrophy in patients with essential hypertension: the Candesartan Assessment in the Treatment of Cardiac Hypertrophy (CATCH) study. J Hypertens, 20:2293-300.

Dahlof B, Pennert K, Hansson L. 1992. Reversal of left ventricular hypertrophy in hypertensive patients: a meta-analysis of 109 treatment studies. Am J Hypertens, 5:95-100. 
Dahlof B. 2001. Left ventricular hypertrophy and angiotensin II antagonists. Am J Hypertens, 14:174-82.

Dahlof B, Zanchetti A, Diez J, et al. 2002. Effect of losartan and atenolol on left ventricular mass and neurohormonal profile in patients with essential hypertension and left ventricular hypertrophy. J Hypertens, 20:1855-64.

Devereux RB, Wachtell K, Gerdts E, et al. 2004. Prognostic significance of left ventricular mass changes during treatment of hypertension. JAMA, 292:2350-6.

Devereux RB, Dahlof B, Gerdts E, et al. 2004. Regression of hypertensive left ventricular hypertrophy by losartan compared to atenolol. Circulation, 110:1456-62.

Disertori M, Latini R, Maggioni AP, et al. 2006. Rationale and design of the GISSI-Atrial Fibrillation Trial: a randomized, prospective, multicentre study on the use of valsartan, an angiotensin II AT1-receptor blocker, in the prevention of atrial fibrillation recurrence. J Cardiovasc Med, 7:29-38.

Ducharme A, Swedberg K, Pfeffer MA, et al. 2006. Prevention of atrial fibrillation in patients with symptomatic chronic heart failure by candesartan in the Candesartan in Heart failure: Assessment of Reduction in Mortality and morbidity (CHARM) program. Am Heart $J, 151: 985-91$.

Fogari R, Mugellini A, Destro M, et al. 2006. Losartan and prevention of atrial recurrence in hypertensive patients. $J$ Cardiovasc Pharmacol, 47:46-50.

Healey JS, Baranchuck A, Crystal E, et al. 2005. Prevention of atrial fibrillation with angiotensin-converting enzyme inhibitors and angiotensin receptor blockers. A meta-analysis. $J$ Am Coll Cardiol, 45:1832-39

Hennersdorf MG, Strauer BE. 2001. Arterial hypertension and cardiac arrhythmias. J Hypertens, 19:167-77.

Kaschina E, Unger T. 2003. Angiotensin AT1/AT2 receptors: regulation, signalling and function. Blood Press, 12:70-88.

Kannel WB, Wolf PA, Benjamin EJ, et al. 1998. Prevalence, incidence, prognosis, and predisposing conditions for atrial fibrillation: populationbased estimates. Am J Cardiol, 82:2N-9N.

Klingbeil AU, Schneider M, Martus P, et al. 2003. A meta-analysis of the effects of treatment on left ventricular mass in essential hypertension. Am J Med, 115:41-6.

Koren MJ, Devereux RB, Casale PN, et al. 1991. Relation of left ventricular mass and geometry to morbidity and mortality in uncomplicated essential hypertension. Ann Intern Med, 114:345-52.

Leonetti G, Cuspidi C. 1995. Heart and vascular changes in hypertension. J Hypertens, 13(Suppl 2):S29-S34.

Levy G, Anderson KM, Savage DD, et al. 1990. Prognostic implications of echocardiographically determined left ventricular mass in the Framingham Heart Study. N Engl J Med, 322:1561-6.

Madrid AH, Bueno MG, Rebollo JMG, et al. 2002. Use of irbesartan to maintain sinus rhythm in patients with long-lasting persistent atrial fibrillation. Circulation, 106:331-6.

Mitchell GF, Vasan RS, Keyes MJ, et al. 2007. Pulse pressure and risk of new onset atrial fibrillation. JAMA, 297:709-15.

Maggioni AP, Latini R, Carson PE, et al. 2005. Valsartan reduces the incidence of atrial fibrillation in patients with heart failure: results from the Valsartan Heart Failure Trial (Val-HeFT). Am Heart $J$, 149:548-57.

Malmqvist K, Kahan T, Edner M, et al. 2000. Regression of left ventricular hypertrophy in human hypertension with irbesartan. $J$ Hypertens, 19:1167-76.

Mancia G, Zanchetti A, Agabiti-Rosei E, et al. 1997. Ambulatory blood pressure is superior to clinic blood pressure in predicting treatmentinduced regression of left ventricular hypertrophy. Circulation, 95:1464-70.

Okin PM, Wacthell K, Devereux RB, et al. 2006. Regression of electrocardiographic left ventricular hypertrophy and decreased incidence of new-onset atrial fibrillation in patients with hypertension. JAMA, 296:1242-8.
Perlini S, Muiesan ML, Cuspidi C, et al. 2001. Midwall mechanics are improved after regression of hypertensive left ventricular hypertrophy and normalization of chamber geometry. Circulation, 103:678-83.

Reudelhuber TL, Bernstein KE, Delafontaine P. 2007. Is angiotensin II a direct mediator of left ventricular hypertrophy? Time for another look. Hypertension, 49:1196-201.

Rogg H, de Gasparo M, Graedel E, et al. 1996. Angiotensin II-receptor sybtypes in human atria and evidence for alterations in patients with cardiac dysfunction. Eur Heart J, 17:1112-120.

Schlaich MP, Schobel H, Langenfeld MRV, et al. 1998. Inadequate suppression of angiotensin II modulates left ventricular structure in humans. Clin Nephrol, 49:153-9.

Schmieder RE. 2005. The role on non-haemodynamic factors in the genesis of LVH. Nephron Dial Transplant, 20:2610-12.

Schmieder RE, Langefeld MR, Friedrich A, et al. 1996. Angiotensin II related to sodium excretion modulates left ventricular structure in human essential hypertension. Circulation, 94:1304-9.

Schmieder RE, Messerli FH, Garavaglia GE, et al. 1988. Does the reninangiotensin-aldosterone system modify cardiac structure and function in essential hypertension? Am J Med, 84(Suppl A):136-39.

Schmieder RE, Martus P, Klingbeil AU. 1996. Reversal of left ventricular hypertrophy in essential hypertension. A meta-analysis of randomized double-blind studies. JAMA, 275:1507-13.

Schmieder RE, Schlaich MF, Klingbeil AU, et al. 1998. Update on reversal of left ventricular hypertrophy in essential hypertension (a meta-analysis of all randomized double-blind studies until December 1998). Nephrol Dial Transplant, 13:564-9.

Schmieder R, Kjeldsen SE, Julius S, et al. 2006. Reduced incidence of new-onset atrial fibrillation with angiotensin II receptor blockade: the VALUE trial [abstract]. J Hypertens, 24:(Suppl)S3.

Schneider MP, Klingbeil AU, Delles C, et al. 2004. Effect of irbesartan versus atenolol on left ventricular mass and voltage. Results of the CardioVascular Irbesartan Project. Hypertension, 44:61-6.

Sega R, Trocino G, Lanzarotti A, et al. 2001. Alterations of cardiac structure in patients with isolated, ambulatory, or home hypertension: data from the general population (Pressione Arteriose Monitorate E Loro Associazioni [PAMELA]) Study. Circulation, 104:1385-92.

Trimarco B, De Luca N, Ricciardelli B, et al. 1988. Cardiac function in systemic hypertension before and after reversal of left ventricular hypertrophy. Am J Cardiol, 62:745-50.

Tsai CT, Lai LP, Lin JL, et al. 2004. Renin-angiotensin system gene polymorphisms and atrial fibrillation. Circulation, 104:2608-14.

Verdecchia P, Porcellati C, Reboldi G, et al. 2001. Left ventricular hypertrophy as an independent predictor of acute cerebrovascular events in essential hypertension. Circulation, 104:2039-44.

Verdecchia P, Angeli F, Borgioni C, et al. 2003. Changes in cardiovascular risk by reduction of left ventricular mass in hypertension: a metaanalysis. Am J Hypertens, 16:895-9.

Verdecchia P, Reboldi G, Gattobigio R, et al. 2003. Atrial fibrillation in hypertension : predictors and outcome. Hypertension, 41:218-23.

Wachtell K, Lehto M, Gerdts E, et al. 2005. Angiotensin II receptor blockade reduces new-onset atrial fibrillation and subsequent stroke compared to atenolol. J Am Coll Cardiol, 45:712-19.

Yasunari K, Maeda K, Watanabe T, et al. 2004. Comparative effects of valsartan versus amlodipine on left ventricular and reactive oxygen species formation by monocytes in hypertensive patients with left ventricular hypertrophy. J Am Coll Cardiol, 43:2116-23. 
